\title{
Microparticle Formation via Tri-needle Coaxial Electrospray at Stable Jetting Modes
}

Yao, Z., Zhang, C., Ahmad, Z., Peng, Y., \& Chang, M. (2020). Microparticle Formation via Tri-needle Coaxial Electrospray at Stable Jetting Modes. Industrial and Engineering Chemistry Research, 59(32), 14423-14432. https://doi.org/10.1021/acs.iecr.0c02677

Link to publication record in Ulster University Research Portal

\section{Published in:}

Industrial and Engineering Chemistry Research

Publication Status:

Published (in print/issue): 12/08/2020

DOI:

10.1021/acs.iecr.0c02677

\section{Document Version}

Author Accepted version

\section{General rights}

Copyright for the publications made accessible via Ulster University's Research Portal is retained by the author(s) and / or other copyright owners and it is a condition of accessing these publications that users recognise and abide by the legal requirements associated with these rights.

\section{Take down policy}

The Research Portal is Ulster University's institutional repository that provides access to Ulster's research outputs. Every effort has been made to ensure that content in the Research Portal does not infringe any person's rights, or applicable UK laws. If you discover content in the Research Portal that you believe breaches copyright or violates any law, please contact pure-support@ulster.ac.uk. 
Microparticle formation via tri-needle coaxial electrospray at stable jetting modes

Zhi-Cheng Yao, ${ }^{\mathrm{a}, \mathrm{b}}$, Chunchen Zhang ${ }^{\mathrm{a}, \mathrm{b}}$, Zeeshan Ahmad ${ }^{\mathrm{c}}$, Yu Peng ${ }^{\mathrm{d}}$, Ming-Wei Chang ${ }^{\mathrm{a}, \mathrm{b}, \mathrm{e}^{*}}$

a Key Laboratory for Biomedical Engineering of Education Ministry of China, Zhejiang University, Hangzhou 310027, PR China

b Zhejiang Provincial Key Laboratory of Cardio-Cerebral Vascular Detection Technology and Medicinal Effectiveness Appraisal, Zhejiang University, Hangzhou 310027, PR China

c The Leicester School of Pharmacy, De Montfort University, The Gateway, Leicester, LE1

9BH, UK

d College of Civil Engineering and Architecture, Zhejiang University, Hangzhou 310027, PR China

e Nanotechnology and Integrated Bioengineering Centre, University of Ulster, Jordanstown Campus, Newtownabbey, BT37 0QB, Northern Ireland, UK.

*corresponding author:

Dr. Ming-Wei Chang

*Email: m.chang@ulster.ac.uk; Tel: + 4428953671 


\begin{abstract}
Multi-layered structured organic particles have had an extensive impact on a wide array of biomedical applications not limited to drug delivery, imaging and biosensing. A trineedle coaxial electrospraying system was utilized to engineer multi-layered polymeric particles in a one-step, facile process at ambient temperatures. The effect of the dominant processing parameters on the development of a conical cusp that eventually ejects an ultrathin liquid ligament were firstly explored here. Subsequently, the validation of the intermediate solutions that possessed different conductivities on stabilizing jetting modes and the resulting particle morphology was also investigated. Polycaprolactone (PCL) solutions with different molecular weights were selected as the outer layer using fluids with various conductivities. Five different formulations were studied as the intermediate layers: PCL in acetic acid, ethyl cellulose in acetic acid, ethyl cellulose in dichloromethane, ethyl cellulose in ethanol and silicone oil and polyvinyl pyrrolidone in water. The results systematically demonstrated that the processing parameters (type of polymer, polymer molecular weight, solution concentration, flow rate, applied voltage and collector distance) play a significant role in the formation of the stable Taylor cone. This study further identified that the coaxial arrangement of three needles successfully produced multi-layered microspheres with uniform size distribution.
\end{abstract}




\section{Introduction}

The engineering and development of organic particles has yielded many opportunities to address and treat many challenging medical conditions. The use of polymers in particulate form has exponentially increased in recent years, especially as contrast agents and sensors. ${ }^{1,2}$. Successful encapsulation of multiple drugs has been of great interest, especially in cases were patient compliance and advanced therapy is crucial. For example, a combination of anti-inflammatory and anti-cancer drugs is promising for cancer therapy as both the inflammation and prevention of further tumor development can be targeted ${ }^{3,4}$. By encapsulating two or more actives in one dosage can deliver synergistic therapeutic effects and reduce unwanted side effects ${ }^{5}$.

Microparticles with various morphologies and functions have attracted widespread attention in biomedical fields. Their uses have extended to serving as drug carriers for oral capsules or injectable agents, providing an adequate environment for 3D cell culture and acting as a platform for multimodal imaging and therapy ${ }^{6-8}$. Functionalities of the particles largely depend on their surface morphology and internal structure ${ }^{9,10}$. Due to the possibilities of fabricating microparticles with unique morphologies, the development of new and complex functionalities has also arisen ${ }^{11,12}$. For example, polymeric hollow particles with pores can be readily used to load actives for an array of applications such as film coatings, drug delivery systems, catalysts, crystals, for chemistry, biotechnology, and materials science ${ }^{13}$. Several methods have been utilized for synthesizing such composite particles however most of these approaches are multistep processes; requiring additional reaction conditions such as inert gas or maintaining reaction temperatures or using elevated processing temperatures or pressure ${ }^{14,15}$. As a result, there are some hurdles when attempting to continuously produce multi-layered particles for in line production. In addition to this, current research demonstrates that 
only polymeric materials with appropriate biosafety, biocompatibility, and biodegradation are considered for particle engineering; limiting the selection of polymers that can be used with these methods. ${ }^{16}$.

The electrospraying technique is a one-step preparation method which has been widely used to engineer particles on micro and nano scale ${ }^{17,18}$. The diameter and surface morphology of resulting particles is regulated by process parameter manipulation (i.e. flow rate, applied voltage and collector distance $)^{19,20}$. Tri-needle coaxial electrospray is a facile and versatile method which has been used to produce multilayered (more specifically tri-layered) polymeric particles in a one step process which is operated at ambient temperature ${ }^{21}$. This engineering method allows particles to be collected with a solvent, proving to be a cost-effective and convenient way of yielding porous polymeric particles. Although the tri-needle electrospraying technique can be exploited in preparing composite structure with multiple layers; stable Taylor cone formation is crucially affected by different parameters and material and/or solution characteristics 22-24. Therefore, in this study, we comprehensively explored fundamental conditions of achieving a stable co-jetting mode during the tri-axial electrospray process by investigating the effects of processing parameters (type of polymer, polymer molecular weight, solution concentration, flow rate, applied voltage and collector distance) on the formation of Taylor cone and its downstream impact on particle size distribution and morphology.

Polycaprolactone (PCL), a FDA approved biomaterial, is a synthetic biocompatible polymer with excellent biodegradability, spinnability, good mechanical properties. It has been widely used to engineer particles with various structures for numerous biomedical applications ${ }^{25}$. Dimethyl silicone oil has also shown its potential in coaxial electrospraying due to its advantageous viscosity and stable chemical properties. Ethyl 
cellulose (EC) and polyvinylpyrrolidone (PVP) can be processed via the multi-needle electrospray technique with PCL due to their immiscibility. In this study, PCL was employed as the inner and outer layers while silicone oil, EC or PVP as the formed the intermediate layer. The effect of polymer molecular weight and the dominant processing parameters on the stability of jetting mode and the morphology of the sprayed products were systematically explored.

\section{Materials and Methods}

\subsection{Materials}

Poly-caprolactone (PCL) with different molecular weights (Mw: 14000, 45000, 80000 $\mathrm{g} / \mathrm{mol}$ ), polyvinylpyrrolidone (PVP) with a molecular weight of $10000 \mathrm{~g} / \mathrm{mol}$, and rhodamine B were obtained from Sigma-Aldrich (St. Louis, USA). Dimethyl silicone oil, ethyl cellulose (EC), and Nile red were purchased from Aladdin Chemistry Co., Ltd (Shanghai, China). Dichloromethane (DCM), ethanol, and glacial acetic acid (HAc) were received from Sinopharm Chemical Reagent Co., Ltd (Shanghai, China). Deionized water (DI water) was produced with a Millipore Milli-Q Reference ultrapure water purifier (USA). All chemicals were utilized without any additional purification.

\subsection{Solution preparation}

Briefly, the polymers were respectively dissolved into corresponding solvents, and placed on a magnetic stirring (VELP ARE heating magnetic stirrer, Italy) in a flask for 24 hours at $300 \mathrm{rpm}$ at room temperature $\left(\sim 25^{\circ} \mathrm{C}\right)$ to achieve homogenous solutions. PCL (with different molecular weights: 14000, 45000, $80000 \mathrm{~g} / \mathrm{mol}$ ) was dissolved in HAc; PVP was dissolved in DI water; EC was dissolved in HAc, ethanol, or DCM. For mapping the jetting status and the formation of a stable Taylor cone during multi-fluid 
processing, fluorescent dyes at concentration of $0.01 \mathrm{w} / \mathrm{v} \%$ were added and stirred uniformly when preparing the middle solution. According to the dye's solubility, PVP solution was stained with rhodamine $\mathrm{B}$, while other polymer solutions were stained with Nile red dye.

\subsection{Characterization of physical liquid properties}

The surface tension of the solutions were measured using an interfacial tensiometer (SK200KB, KINO Industry Co. Ltd., USA) based on pendant-drop method, using Equation $1^{26,27}$ :

$$
\gamma=\frac{g D_{e}^{2} \Delta \rho}{H}
$$

(Equation 1)

where $\gamma$ is the surface tension of the solution, $\Delta \rho$ is the density difference between the solution and air, $D_{e}$ is the maximum diameter of the hanging droplet, $H$ is the correction coefficient, which relates to the shape of droplet.

Solution density was measured using a pycnometer and Equation 2:

$$
\rho_{\mathrm{x}}=\frac{\mathrm{m}_{\mathrm{x}}-\mathrm{m}_{0}}{\mathrm{~V}_{0}}+\rho_{0}
$$

where $V_{0}$ is the volume of the bottle, $\rho_{0}$ is the density of pure water, $\rho_{x}$ is the density of the testing solution, $\mathrm{m}_{0}$ is the total mass of the bottle filled with water, $\mathrm{m}_{\mathrm{x}}$ is the total mass of the bottle filled with testing solution.

The viscosity of the polymeric solutions was measured using a viscometer (LVDV-II, Brookfield, USA). $2 \mathrm{~mL}$ of solution sample was added into stainless steel wells and the viscosity was measured at $25^{\circ} \mathrm{C}$, using a S21 spindle at $140 \mathrm{rpm}$. Electrical conductivity was measured with a YSI 3200 conductivity meter (YSI, USA) at $25^{\circ} \mathrm{C}$.

\subsection{Fabrication of multilayered composite particles}


The multilayered microparticles were engineered using a tri-needle electrospraying apparatus. As shown in Figure 1, the electrospraying system here consists of 3 coaxially arranged processing spinneret, a high voltage supply (Glassman high voltage Inc. series FC, USA), three syringe pumps (KD Scientific KDS100, USA), a chargecoupled devices (CCD) camera (Baumer TXG02C, Germany), and a grounded ringshape electrode. The inner and outer diameters of the concentric needles were $0.50 \mathrm{~mm}$ and $0.31 \mathrm{~mm}, 1.60 \mathrm{~mm}$ and $1.07 \mathrm{~mm}$, and $2.85 \mathrm{~mm}$ and $2.26 \mathrm{~mm}$, respectively for the inner, middle and outer needle.

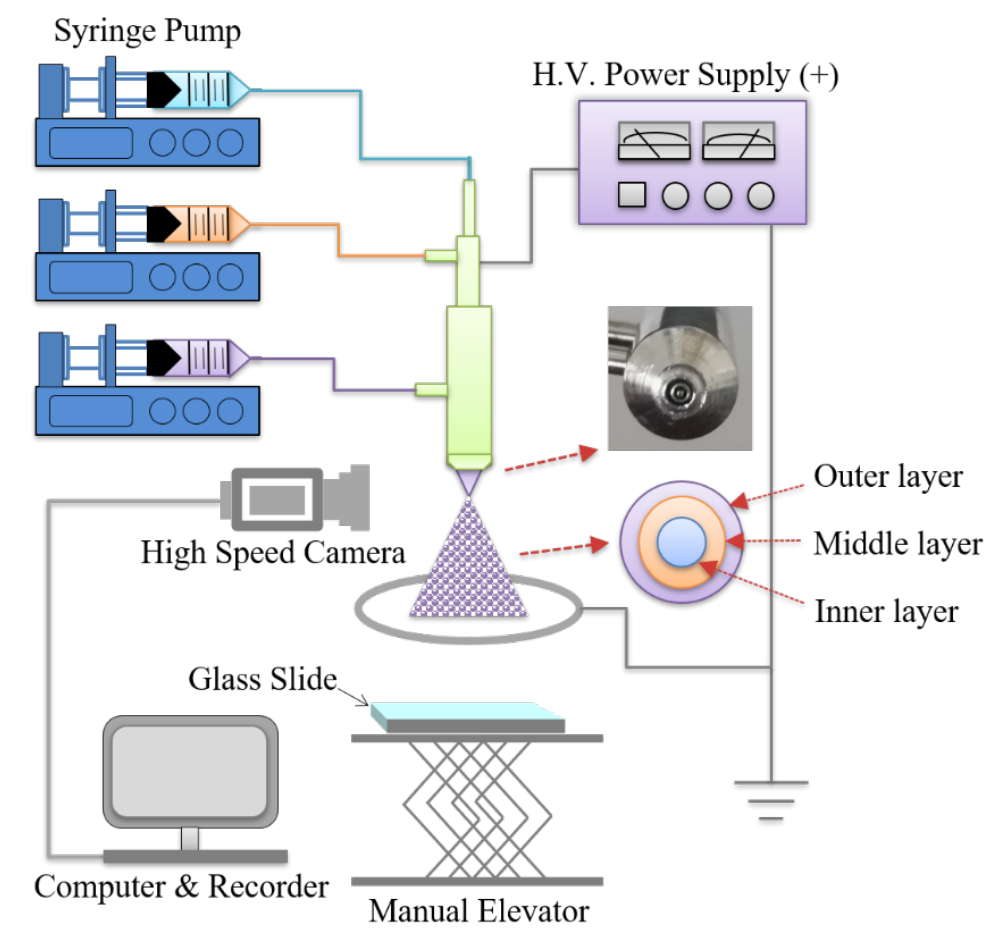

Figure 1. Schematic diagram of the tri-axial electrospraying system.

Solutions were drawn into $5 \mathrm{ml}$ plastic syringes which were attached to the conductive needle tip using silicone tubing. The infusion rate was controlled using high-precision programmable syringe pumps. An electric field was introduced (maximum $\sim 30 \mathrm{kV}, 0.1$ $\mathrm{mA}$ ) between the tri-axial needle and ground electrode via a high voltage supply (Glassman high voltage Inc. series FC, USA). The ring-shape electrode with inner and 
outer diameters of $2.7 \mathrm{~cm}$ and $3.5 \mathrm{~cm}$ was set directly below the tri-axial needle $5 \mathrm{~mm}$ away.

For investigating the influence of processing parameters, the collector distance was kept at $20 \mathrm{~cm} .20 \mathrm{w} / \mathrm{v} \%$ PCL dissolved in HAc was infused into the outer layer of the spinneret, when dimethyl silicone oil and $10 \mathrm{w} / \mathrm{v} \%$ PCL solution were loaded into the middle and inner layers, respectively. The flow rates of the outer, middle and inner solutions were kept at $5.0,1,5$, and $0.8 \mathrm{~mL} / \mathrm{h}$, respectively while the applied voltage was kept at $12 \mathrm{kV}$. During the electrospraying process, the coaxially arranged set-up was observed using a high-speed camera.

\subsection{Particles morphology observation}

Optical microscopy (OM, Pheonix BMC503-ICCF, China) and field emission scanning electron microscopy (SEM, ProX, Phenom, Netherlands) were used to study the size and surface morphology of the resulting particles. For SEM analysis, samples were placed on a metallic stud using double-sided conductive tape and were subsequently sputtered coating with a layer of gold under vacuum (Ion sputter MC 1000, Hitachi, Japan) for $90 \mathrm{~s}$ at a current intensity of $25 \mathrm{~mA}$. The micrographs were subsequently analyzed using ImageJ software (National Institute of Health, USA) to measure the mean particle diameter. The average diameter of the engineered particles was quantified 100 randomly sampled particles. All the statistical graphs were plotted using Origin software (OriginLab, USA). All the experiments were performed in triplicate and data is presented as mean \pm standard deviation $(n=3)$.

\section{Results and Discussion}

\subsection{Jetting mode deformation upon altered voltage}


A stable Taylor Cone is a pre-requisite for generating uniform electrosprayed particles ${ }^{28}$. Here, $20 \mathrm{w} / \mathrm{v} \%$ PCL $(\mathrm{Mw}=45000 \mathrm{~g} / \mathrm{mol})$ was dissolved in HAc and injected into the outer layer of the spinneret, when silicone oil and $10 \mathrm{w} / \mathrm{v} \%$ PCL solution were infused into the intermediate and inner needles of the spinneret, respectively. The flow rates of the outer, middle and inner solutions were kept at $5,1.5$, and $0.8 \mathrm{~mL} / \mathrm{h}$, respectively. As shown in Figure 2, increasing the applied voltage from $0 \mathrm{kV}$ to $20 \mathrm{kV}$, the droplet at the spinneret tip transitioned from dripping mode (Figure 2a-2g) to stable jetting (Figure 2h-2n) and finally to multi-jetting mode (Figure 2o).
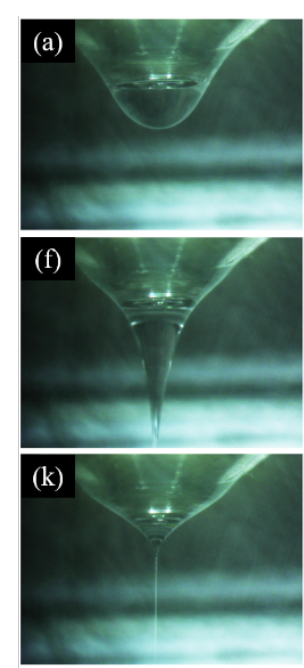
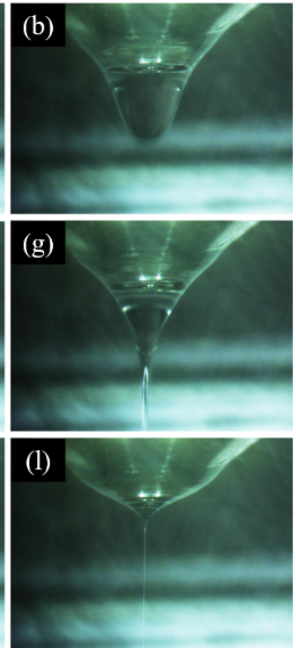
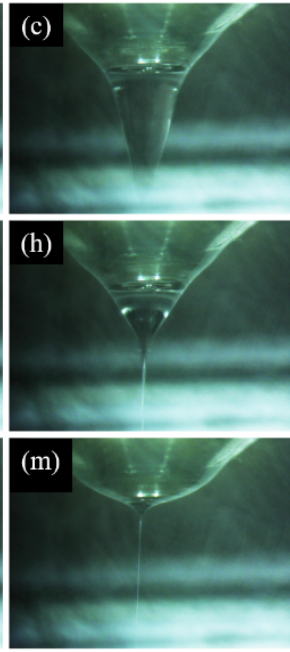
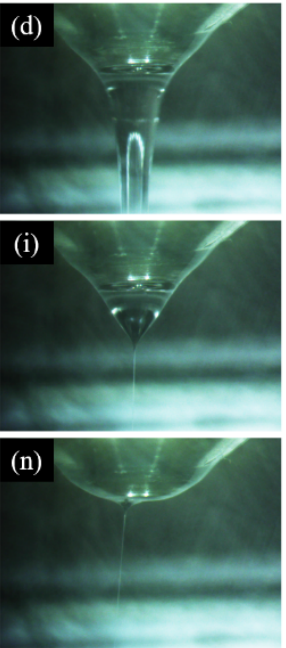
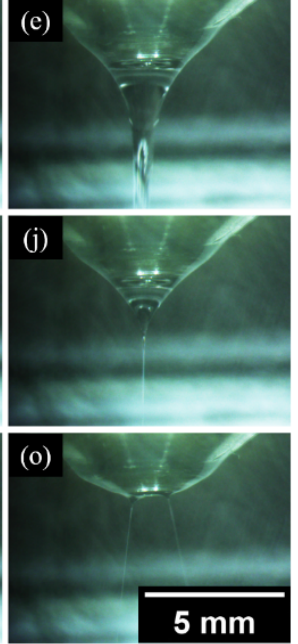

Figure 2. Transformation of the Taylor cone during the electrospraying process by increasing the applied voltage from 0 to $20 \mathrm{kV}$. A stable cone jet was achieved at 12 $\mathrm{kV}$, as indicated in (Figure 2k); multi-jet occurred when increasing applied voltage to $20 \mathrm{kV}$, as shown in (Figure 2o).

\subsection{Effect of molecular weight of shell solution on jet flow and particle formation}

The outer solution was the 'driving liquid' during the coaxial electrospraying process, due to the higher electric conductivity of the PCL solution $(0.054 \mu \mathrm{S} / \mathrm{cm})$ compared with the intermediate liquid (silicone oil) (as shown in Table 1). Hence, the outer PCL solution becomes the dominating force in the electrospraying process ${ }^{29}$. As mentioned 
earlier, to achieve uniform particles a stable cone-jet must be attained. The formation of this stable jet is synthetically influenced by gravity, applied electrical force and solution surface tension ${ }^{30}$. Liquid surface tension was significantly affected by molecular weight and concentration of the polymers. Hence, three different PCL molecular weights were used for the outer solution to investigate the effect of molecular weights of the outer polymeric solution on jetting mode and the resulting particle size distribution. Here, silicone oil and $10 \mathrm{w} / \mathrm{v} \%$ PCL $(\mathrm{Mw}=45000 \mathrm{~g} / \mathrm{mol})$ solution were used as the intermediate and inner layer, respectively.

Table 1. Physical properties of solutions

\begin{tabular}{|c|c|c|c|c|c|}
\hline Solute@Solvent & $\begin{array}{l}\text { Concentration } \\
(\mathrm{w} / \mathrm{v} \%)\end{array}$ & $\begin{array}{l}\text { Density } \\
\left(\mathrm{g} / \mathrm{cm}^{3}\right)\end{array}$ & $\begin{array}{l}\text { Conductivity } \\
(\mathrm{uS} / \mathrm{cm})\end{array}$ & $\begin{array}{l}\text { Surface } \\
\text { Tension } \\
(\mathrm{mN} / \mathrm{m})\end{array}$ & $\begin{array}{l}\text { Viscosity } \\
(\mathrm{mPa} \cdot \mathrm{s})\end{array}$ \\
\hline EC@HAc & 20 & 1.064 & 0.330 & 42.052 & $8454 \pm 29$ \\
\hline EC@DCM & 20 & 1.275 & 0.358 & 46.365 & $3839 \pm 76$ \\
\hline EC@EtOH & 10 & 0.826 & 20.570 & 34.783 & $1727 \pm 4$ \\
\hline PVP@Water & 20 & 1.035 & 1133.000 & 98.676 & $44 \pm 2$ \\
\hline Silicone Oil & I & 0.970 & 0.00 & 21.846 & $582 \pm 2$ \\
\hline PCL@HAc & 10 & 1.050 & 0.053 & 43.033 & $59 \pm 1$ \\
\hline \multicolumn{6}{|l|}{$\mathrm{Mw}=14000 \mathrm{~g} / \mathrm{mol}$} \\
\hline PCL@HAc & 10 & 1.055 & 0.208 & 39.630 & $1136 \pm 4$ \\
\hline \multicolumn{6}{|l|}{$\mathrm{Mw}=80000 \mathrm{~g} / \mathrm{mol}$} \\
\hline PCL@HAc & 5 & 1.049 & 0.054 & 41.687 & $43 \pm 3$ \\
\hline \multicolumn{6}{|l|}{$\mathrm{Mw}=45000 \mathrm{~g} / \mathrm{mol}$} \\
\hline PCL@HAc & 10 & 1.053 & 0.051 & 31.606 & $133 \pm 1$ \\
\hline \multicolumn{6}{|l|}{$\mathrm{Mw}=45000 \mathrm{~g} / \mathrm{mol}$} \\
\hline PCL@HAc & 20 & 1.057 & 0.045 & 39.646 & $1002 \pm 3$ \\
\hline $\mathrm{Mw}=45000 \mathrm{~g} / \mathrm{mol}$ & & & & & \\
\hline
\end{tabular}


As seen in Figure 3a-c, by simultaneously regulating the flow rate and applied voltage, a stable jet could be achieved using the parameters highlighted in green. The most versatile solution which achieved a large operating window when obtaining a stable jet contained PCL with molecular weight of $45000 \mathrm{~g} / \mathrm{mol}$ (Figure 3b). The particles fabricated using this solution exhibited the largest diameter amongst the three groups of different PCL molecular weight (Figure 3g). Figure S1 shows the optical and SEM images of particles generated with different PCL molecular weights. At low molecular weights (14000 and $45000 \mathrm{~g} / \mathrm{mol})$, insufficient particle solidification was observed with oil leakage which subsequently resulted in defective particle structure. Figure 3f shows that electrosprayed particles containing a high molecular weight $(80000 \mathrm{~g} / \mathrm{mol}) \mathrm{PCL}$ shell successfully encapsulated the silicone oil whilst demonstrating the smallest average diameter $(49.29 \pm 13.21 \mu \mathrm{m})$. Some fibrous structures can also be observed (Figure S2). These results demonstrate that the molecular weight of the outer solution significantly affects the jetting mode and subsequently plays an important role in particle morphology and size distribution.

As previously reported ${ }^{31,32}$, during single-needle electrospray, molecular weight of the solution influences the jetting status as shown in Equation 3:

$$
\left(\mathrm{n}_{\mathrm{e}}\right)_{\text {solution }}=\frac{\left(\varphi_{\mathrm{p}} \mathrm{M}_{\mathrm{w}}\right)}{\mathrm{M}_{\mathrm{e}}} \quad(\text { Equation } 3)^{31,32}
$$

Here, $\left(\mathrm{n}_{\mathrm{e}}\right)_{\text {solution }}$ is the entanglement number of the polymer solution, $\varphi_{\mathrm{p}}$ is the volume of the polymer, $M_{w}$ means molecular weight, and $M_{e}$ presents the molecular weight of chain entanglement. $\left(n_{e}\right)_{\text {solution }}$ is proportional to the value of $M_{w}$.

With increasing $\left(n_{e}\right)_{\text {solution }}$, the electrosprayed products transformed from microparticles to particulate/fibrous matrixes and finally fibers. In accordance with this 
both particles and fibers were observed using high molecular weight PCL. Based on this, in order to achieve a stable jetting mode and uniformity of solely particles, PCL with molecular weight of $45000 \mathrm{~g} / \mathrm{mol}$ was selected for the following study.
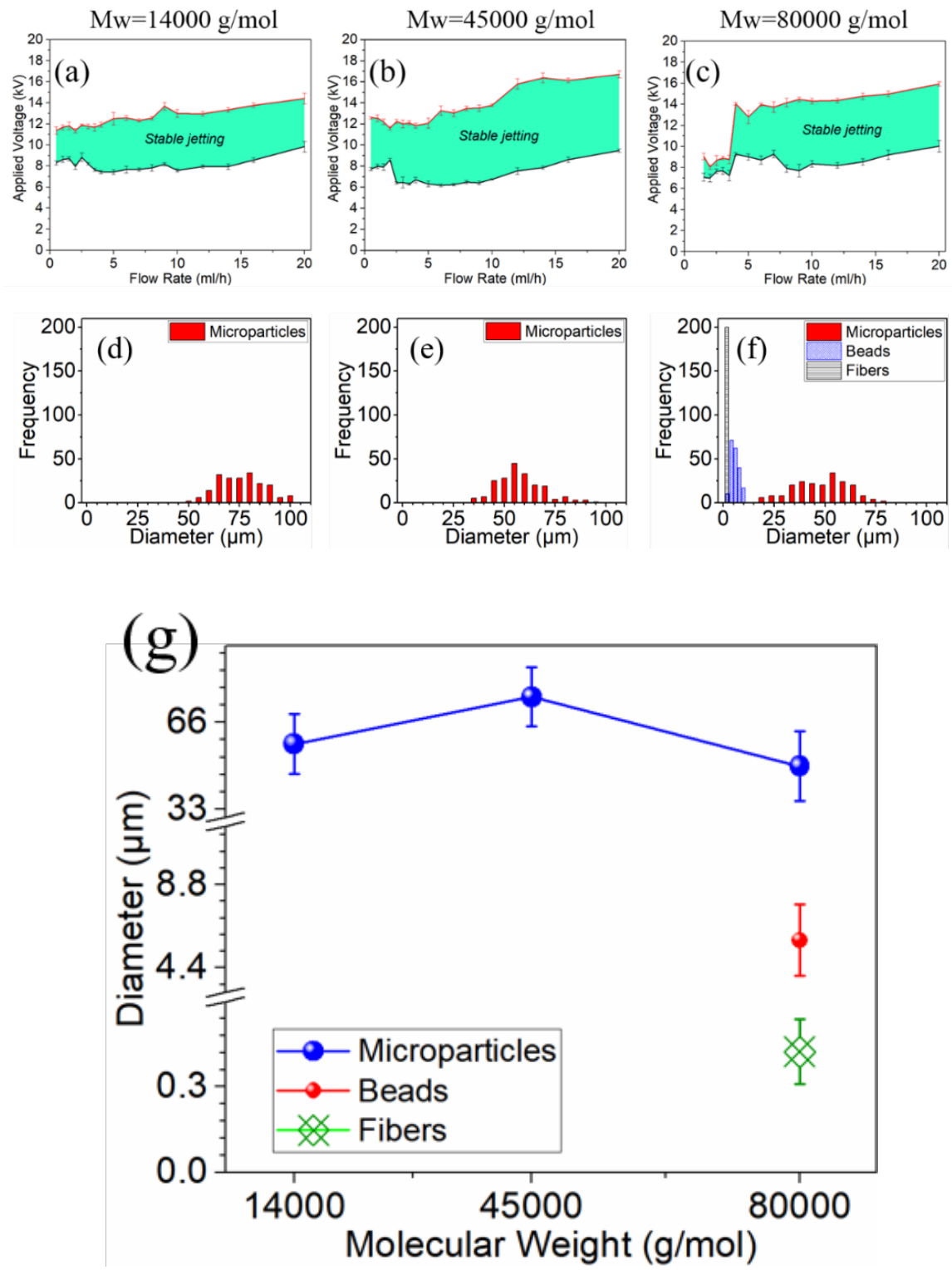

Figure 3. Effect of molecular weight of shell solution on the jet flow and fabricated composite particles. (a)-(c) present the stable jetting range at different process parameters using PCL with molecular weight of 14000, 45000, and $80000 \mathrm{~g} / \mathrm{mol}$ (solution concentration is $20 \mathrm{w} / \mathrm{v} \%$ for all the groups). (d)-(f) show the size distribution of microparticles prepared with PCL solution at molecular weight of 14000, 45000, and $80000 \mathrm{~g} / \mathrm{mol}$, respectively. (g) diameter change as a function of PCL molecular weight. 


\subsection{Effect of shell solution concentration on jet flow and particle formation}

The effect of shell solution concentration on jet mode and particle fabrication was assessed by altering the outer PCL solution concentration from 5 to $20 \mathrm{w} / \mathrm{v} \%$. Here, silicone oil formed the middle layer and $10 \mathrm{w} / \mathrm{v} \%$ PCL formed the inner layer of the resulting particles. Stable jetting mode is defined here as a formation of steady Taylor cone in the absence of multi-jetting.

Figure 4a-c demonstrates a stable jet could be formed using the parameters within the green regions. This competent area of achieving a stable jet amplified with increasing polymer concentration, reaching the largest area at a concentration of $20 \mathrm{w} / \mathrm{v} \%$. This high concentration results in increased polymer chain entanglement subsequently causing an increase in solution viscosity from $43 \mathrm{mPa} . \mathrm{s}(5 \mathrm{w} / \mathrm{v} \% \mathrm{PCL})$ to $1002 \mathrm{mPa} \cdot \mathrm{s}$ (20 w/v\% PCL). As shown in Figure 4d-f, with increasing outer PCL concentration, the diameter distribution of the resulting particles decreased; changing from being between $25-270 \mu \mathrm{m}$ when using $5 \mathrm{w} / \mathrm{v} \%$ PCL to $85-110 \mu \mathrm{m}$ when using $20 \mathrm{w} / \mathrm{v} \%$ PCL. Although these particles were generated by using tri-axial electrospraying, this phenomenon has been observed when using single needle electrospraying. This is thought to be because of the increase in solution concentration and how this allows for sufficient solvent evaporation and solidification of particles ${ }^{33,34}$. The electrosprayed samples when using 5\% w/v PCL seem to be insufficiently dried droplets of nonspherical shape (Figure S2a, d, g, j). Increasing PCL concentration to $10 \mathrm{w} / \mathrm{v} \%$, microparticles were successfully fabricated however once collected the shape of the particles changed to show a more flattened morphology as a result of insufficient collection distance to allow complete solvent evaporation resulting in oil leakage 
(Figure S2b e, h, k). When the concentration was further increased to $20 \mathrm{w} / \mathrm{v} \%$, spherical particles were obtained (Figure S2c, f, i, l). Whilst the theory of sufficient solvent evaporation causing reduced diameter distribution has been substantiated in previous studies, here the decrease in particle diameter could also be attributed to the successful encapsulation of the inner solutions at high shell concentrations. At low concentrations ( 5 and $10 \mathrm{w} / \mathrm{v} \%$ ), polymer entanglement between molecular chains was too weak to effectively encapsulate the intermediate and inner solutions.
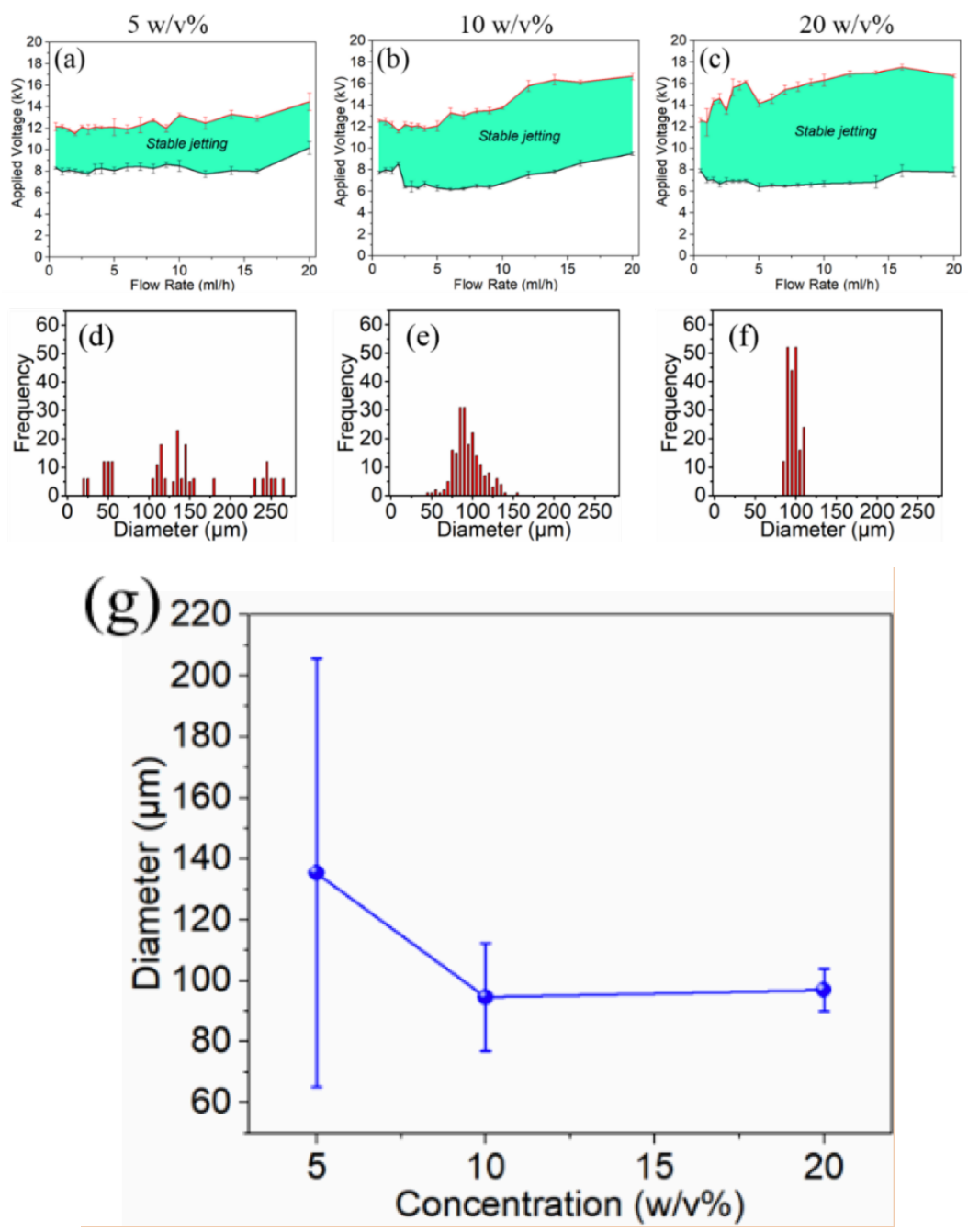

Figure 4. Effect of shell solution concentration on jet flow and the fabricated composite particles. (a)-(c) present the stable jetting range at different processing parameters using PCL at the concentration of 5, 10 and $20 \mathrm{w} / \mathrm{v} \%$, respectively. (d)-(f) show the size 
distribution of microparticles prepared with different PCL concentration. (g) Diameter change as a result of outer PCL solution.

\subsection{Effect of process parameters on jet flow and particle formation}

A multitude of process parameters have been proven to affect the electrospraying process and the fabrication of particles. The most crucial parameters that have been identified are applied voltage, flow rate and working distance (the distance between the needle exit and the collection plate).

The voltage that is applied to the system plays an important role in regulating the jet. The applied voltage must be strong enough to overcome the surface tension of the solution being processed and by atomizing the liquid, the emitted droplet size can be altered/controlled ${ }^{35}$. Here, the voltage was maintained within the range from 9 to 18 $\mathrm{kV}$ with working distance being kept constant at $20 \mathrm{~cm}$. The flow rate of outer, middle and inner solutions was kept at 5.0, 1.5 and $0.8 \mathrm{~mL} / \mathrm{h}$, respectively. The resulting particles were observed using optical microscopy and SEM, as shown in Figure 5. Operating the tri-axial electrospraying process at $9 \mathrm{kV}$ proved to be insufficient due to the fabrication of solvent-rich structures; suggesting incomplete solvent evaporation and inefficacious jetting mode. Upon hitting the collector plate, the impact force caused the structures to flatten (Figure 5a, e, i, $\mathbf{m}$ ), resulting in the leaking of the silicone oil and inner PCL solution. Increasing the voltage to $12 \mathrm{kV}$ (Figure 5n), $15 \mathrm{kV}$ (Figure 5o) and $18 \mathrm{kV}$, the topography of the particles became more prominent; becoming more defined, forming solid spherical structures when operating the process at $18 \mathrm{kV}$ (Figure $5 \mathbf{p})$. 

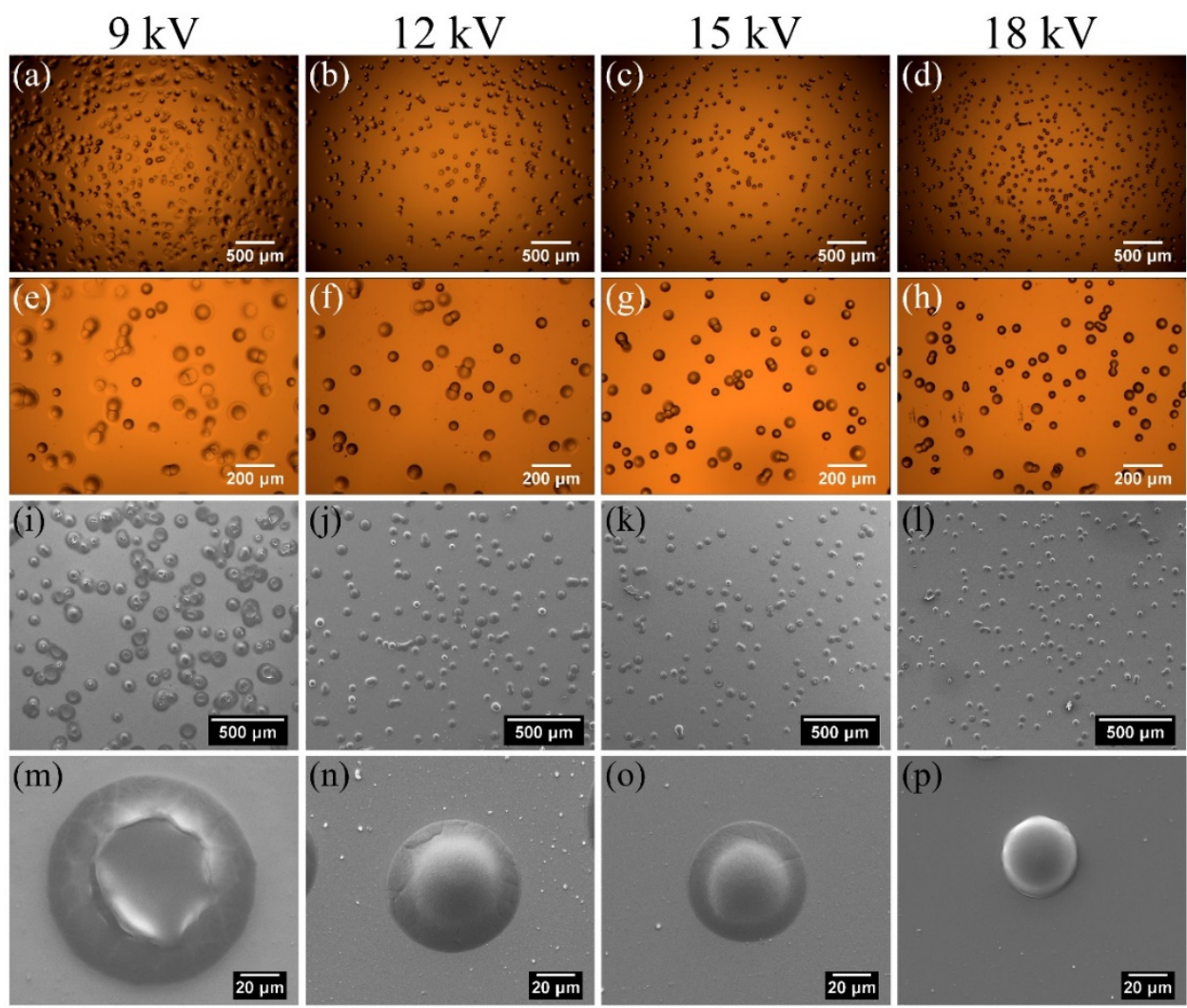

Figure 5. Microparticles prepared using PCL solutions at different applied voltages.

From left to right, the collector distances were $9,12,15,18 \mathrm{kV}$, respectively. The top two lines (a-h) are the optical images presenting the particles size distribution, where the second row is zoom-in version of the first row. (i-p) are the SEM images, showing the surface morphology of the particles, where the bottom row of SEM images is a respective magnified view of electrosprayed particles shown above

Figure 6a-d shows the effect of applied voltage on particle diameter and size distribution. A much narrower diameter distribution was observed at higher applied voltage compared to when operating at a lower voltage $(9 \mathrm{kV})$. It is clear to see from the SEM images and Figure 6 that the collapsed structures seen at lower operating voltages gradually disappeared with increase in voltage. The morphology of the resulting particles transitioned from hemisphere structure to fully spherical particles 
with smaller particle diameters. This variation trend can be seen in Figure $6 \mathbf{m}$. The average particle diameter reduced from $91.6 \pm 30.3 \mu \mathrm{m}$ to $33.1 \pm 16.3 \mu \mathrm{m}$ when increasing the operating voltage from $9 \mathrm{kV}$ to $18 \mathrm{kV}$, respectively. The applied voltage has a significant impact on jetting behavior; increasing voltage results in thinner electrohydrodynamic jet subsequently fabricating smaller droplets due to an enhanced stretching force.
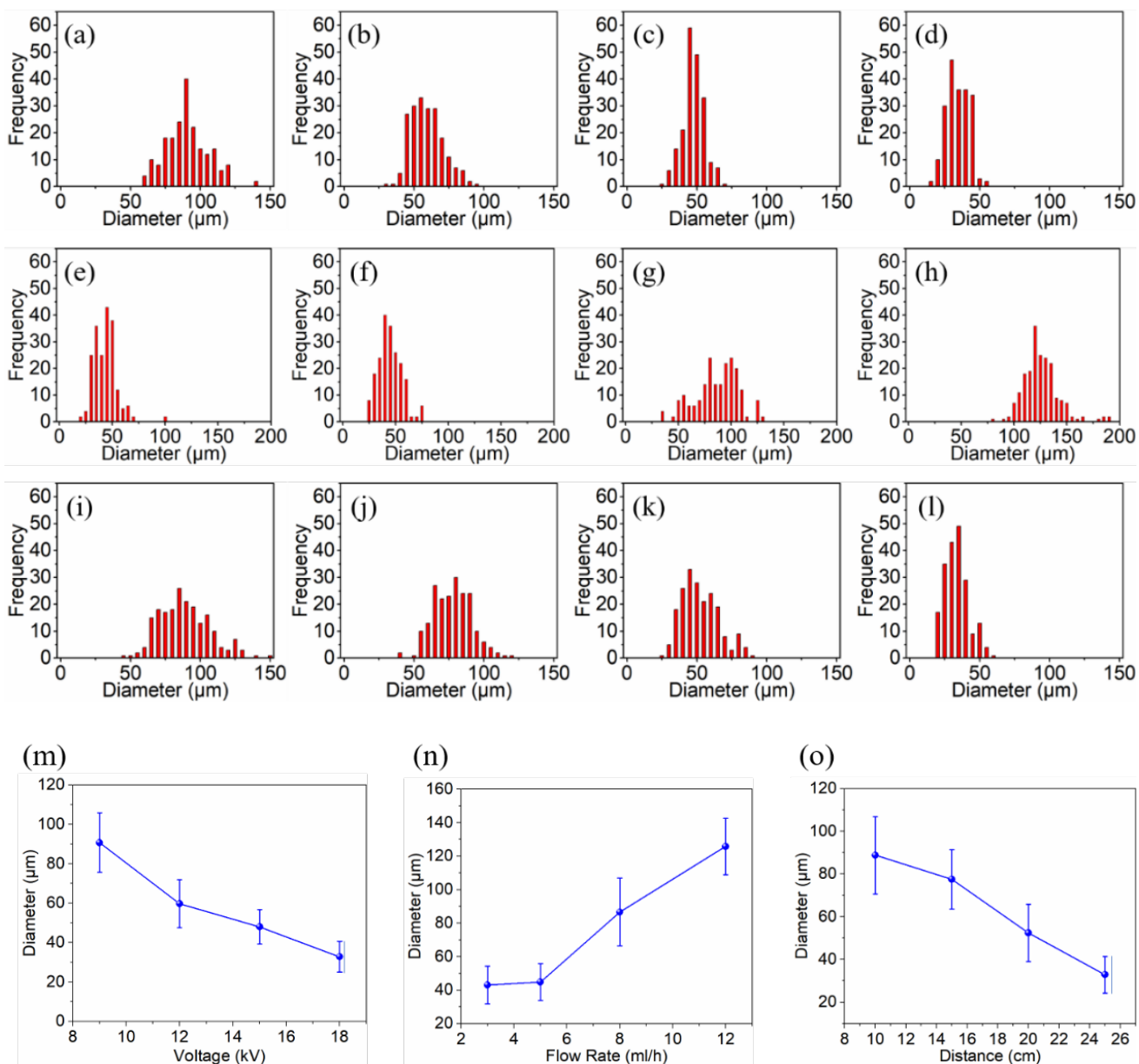

(n)

(o)
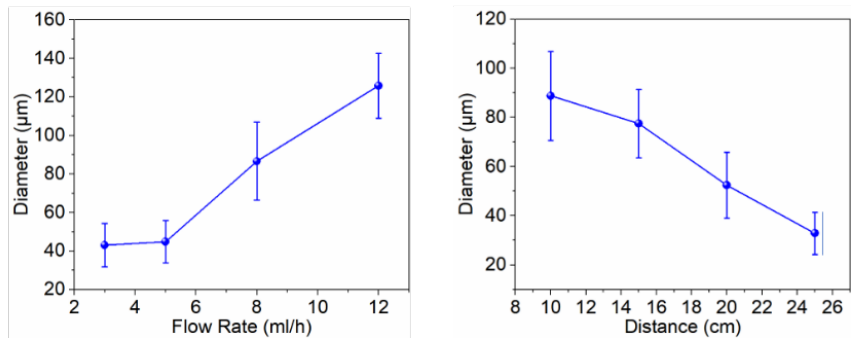

Figure 6. Effects of process parameters on particle size distribution. (a)-(d) Size distribution of microparticles prepared with the applied voltage at $9,12,15$, and $18 \mathrm{kV}$, respectively (flow rate was kept as 5.0, 1.5 and $0.8 \mathrm{~mL} / \mathrm{h}$ for the outer, middle and inner layer, individually); (e)-(h) Size distribution of microparticles prepared at flow rates of 3, 5, 8 and $12 \mathrm{~mL} / \mathrm{h}$; (i)-(l) Size distribution of microparticles prepared with the collector distance at 10, 15, 20, and $25 \mathrm{~cm}$. Diameter change tendency of the particles 
with regulating the parameters: $(\mathrm{m})$ applied voltage; (n) flow rate of the outer PCL solution; (o) collector distance.

To study the effect of flow rate, the outer PCL solution flow rate was varied between 3 and $12 \mathrm{~mL} / \mathrm{h}$ while keeping PCL molecular weight constant at $45000 \mathrm{~g} / \mathrm{mol}$. The intermediate liquid (silicone oil) and inner liquid (10 w/v\% PCL) were infused at a flow rate of $1.5 \mathrm{~mL} / \mathrm{h}$ and $0.8 \mathrm{~mL} / \mathrm{h}$, respectively. $3 \mathrm{~mL} / \mathrm{h}$ proved to be an excessively low flow rate for the shell solution which was ineffective to provide enough liquid to encapsulate the inner oil (Figure S3a, e, i, m). It was found that spherical particles could be achieved when flow rate of the shell solution was increased to $5 \mathrm{~mL} / \mathrm{h}$. The outer solution successfully encapsulated the inner material effectively, fabricating wellstructured microspheres with no oil leakage (Figure S3b, $\mathbf{f}, \mathbf{j}, \mathbf{n}$ ). By further increasing the flow rate of the shell solution to 8 and $12 \mathrm{~mL} / \mathrm{h}$, it was found that the solvent in the outer fluid could not volatilize resulting in deformed, flat particles on the collector plate (Figure S3o and p).

Flow rate optimization for internal and external liquids in co-axial electrospraying is crucial when needing to preparing core-shell particles. Diameter distribution of the resulting particles fabricated using various shell solution flow rates is shown in Figure 6e-h with the variation trend being shown in Figure 6n. It was deduced that increasing the flow rate above $5 \mathrm{~mL} / \mathrm{h}$ led to an increase in microparticle diameter. This is most likely due to the larger volume of solution being infused into the processing system resulting into insufficient atomization and solvent evaporation. A similar trend was also observed at $3 \mathrm{~mL} / \mathrm{h}$; due to the relatively low velocity, the outer fluid failed to effectively encapsulate the middle and inner layers resulting in loss of the silicone oil. 
This subsequently resulted in increased particle diameter due to an enlarged core diameter and a relatively thinner shell under steady co-axial flow. Therefore, the outer flow rate was maintained at a higher flow rate than the inner to ensure sure the core could be sufficiently coated with the outer polymer shell [27]. Ding et al. have previously reported the applied voltage correlation between spraying nozzle and the electrode when engineering PCL microparticles [32].

In the present study, the effect of collection distance (distance from needle exit to ringshaped ground electrode) on PCL core-shell microparticle fabrication using tri-axial electrospraying. The microparticles were prepared using different working distances: $10,15,20$, and $25 \mathrm{~cm}$ while the applied voltage was fixed at $12 \mathrm{kV}$. At the collector distance of $10 \mathrm{~cm}$, incomplete solvent evaporation was observed; resulting in significant oil leakage causing the electrosprayed structures to collapse rendering 10 $\mathrm{cm}$ too short an operating distance. Increasing the working distance to $15 \mathrm{~cm}$ and 20 $\mathrm{cm}$, the resulting particles seem to acquire more spherical contours; with the impact force causing the shape to change and represent hemispherical morphologies. Further increasing the distance to $25 \mathrm{~cm}$ saw the fabrication of fully solidified particles, with a significant decrease in diameter, as shown in Figure S4.

Figure 6i-6l demonstrates that increasing the working distance from 10 to $25 \mathrm{~cm}$ leads to a change in diameter distribution. As the distance increases, the average diameter of the engineered particles decreased with the particle size distribution also narrowing. This may be attributed to the volatile solvent being given sufficient distance to evaporate before particle deposition on the collector plate; resulting in fully formed, dry spherical microparticles. 
Figure 6o highlights the relationship between the mean diameter and the collection distance. When the increasing the working distance from $10 \mathrm{~cm}$ to $25 \mathrm{~cm}$, the mean particle size reduced from $89.5 \pm 41.9 \mu \mathrm{m}$ to $27.3 \pm 19.8 \mu \mathrm{m}$, respectively. The decrease in particle size can be explained by a reduction in incomplete solvent evaporation (as the electrode distance increases); possibly resulting in particles with residual solvent which had not fully evaporated before reaching the collector.

\subsection{Effect of the intermediate solution on the flow rate and microparticle formation}

Compared with the co-axial electrospraying approach, microparticles prepared via triaxial electrospraying contains 3 distinct layers: an inner core and an outer shell with an additional intermediate layer which is often designed to encapsulate bioactive components. It is important to note that the middle layer can affect the particle fabrication process. As a result, the effect of the composition of the intermediate layer (PCL@HAc, EC@HAc, EC@DCM,EC@EtOH, silicone oil andPVP@water) on the stability of the electro-jet and subsequent particle formation.

Jetting modes were recorded between the voltage range of $0-16 \mathrm{kV}$. Dripping, transition, and stable jetting modes are shown in Figure 7. All compositions of the intermediate layer except PVP@water demonstrated a stable jetting mode between 10 and 16 kV; showing good compatibility between all three layers of the multi-layered particles. When operating the electrospraying process when using PVP@water as the middle solution, stable jetting mode could only be achieved using a narrower voltage range; 10 - $14 \mathrm{kV}$. Using a higher voltage than this resulted in solution solidification at the processing needle exit. This may be caused by small sprayed droplets forming at the high voltage, expediting solvent evaporation and especially with increased PCL 
concentration, a thin film formed in the outer layer of the particles inhibiting the formation of a stable jetting.

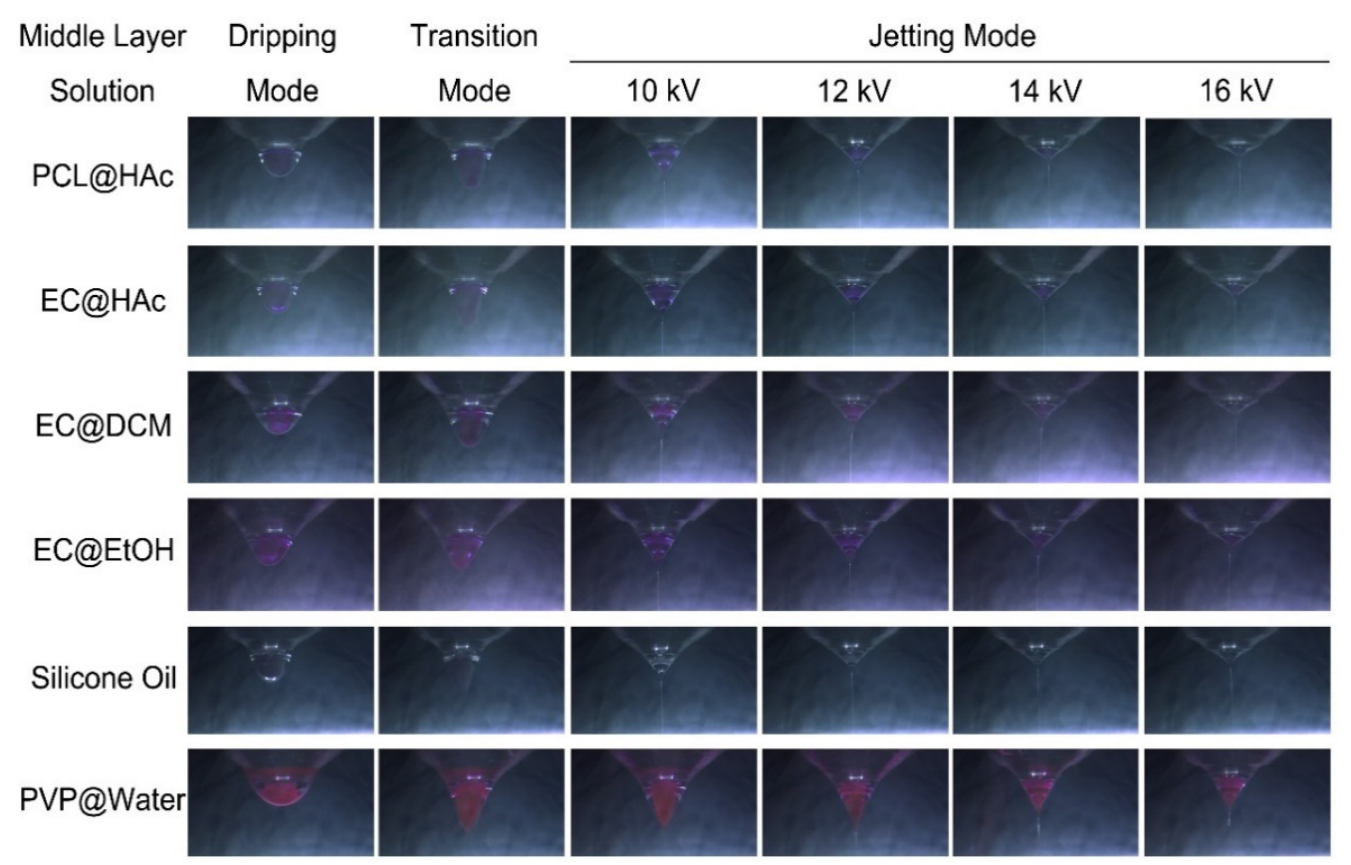

Figure 7. Effect of different intermediate solutions on Taylor cone formation during the tri-needle electrospraying process. As shown in the bottom row, PVP was stained with Rhodamine B, while for other combinations, polymer solutions incorporated staining with Nile red to distinguish distinct layers during the electrospray process.

The results collated here prove that when no significant solidification occurred with the inner and outer solutions, regardless of miscibility characteristics (i.e. PCL@HAc or silicone oil), a stable jet could be formed during tri-axial electrospraying process. While solidification happened either in the inner and outer layers, the jetting stability was found to be seriously affected; as seen when using PVP@Water as the middle layer solution. When there was no chemical interaction among the three solutions in the any of the three layers, the jetting stability is mainly dependent on the reaction among the 
solvents in each layer with each solute. If there is some miscibility between the components within each solution, fluently stable jetting mode would be easily achieved.

As shown in Figure 8, when using dye rhodamine B to label the middle layer when PCL@HAc or EC@HAc was utilized, uniform microspheres were prepared. Here, the solutions in the inner/middle/outer layers were miscible and the fluorescent easily dispensed to the inner and outer layers from the middle layer. Therefore, no solid boundary was found among the three layers. While using EC@EtOH as the middle layer, ultrafine fibers co-occurring with the particles was observed. This was mainly due to the poor solubility of PCL in EtOH. When PCL dispersed from the middle layer to the inner and outer layers, PCL concentration increased leading to the formation of fibrous structures. Additionally, due to good electroconductivity $(20.57 \mu \mathrm{S} / \mathrm{cm})$ of the $\mathrm{EC} / \mathrm{EtOH}$ solution, charging occurred at high applied voltage, facilitating the wide diameter distribution. When using EC@DCM as the middle layer, its conductivity $(0.385 \mu \mathrm{S} / \mathrm{cm})$ was higher than that of the inner $(0.051 \mu \mathrm{S} / \mathrm{cm})$ or outer layers $(0.045$ $\mu \mathrm{S} / \mathrm{cm})$. The driving liquid in the tri-axial electrospray process dominates particle formation. Due to the low boiling point of DCM, it evaporated rapidly, enhancing the solidification of the middle layer. However, non-uniform beaded fibers were obtained, as a result of protracted solidification in the outer layer. When PVP@water was selected as the middle layer, due to the blockage and unstable multi-jetting at the exit of spinneret, amorphous products were collected, including beaded fibers and particles with wide size distribution.

Leakage of the red dye (rhodamine B) identifying the middle layer also indicates the deformation of distinct tri-layered structures caused by the unstable jetting. In contrast, when silicone oil was selected as the middle layer, the products were uniform microspheres, with red marked middle layer, allowing the identification of definitive 
core-shell structures with three layers. These results confirm that although jetting stability and jetting ability is dependent on a combination of interactions concerning gravity, electrical force and interfacial surface tension; the size and morphology of particles can be carefully controlled by modifying or altering various process parameters.

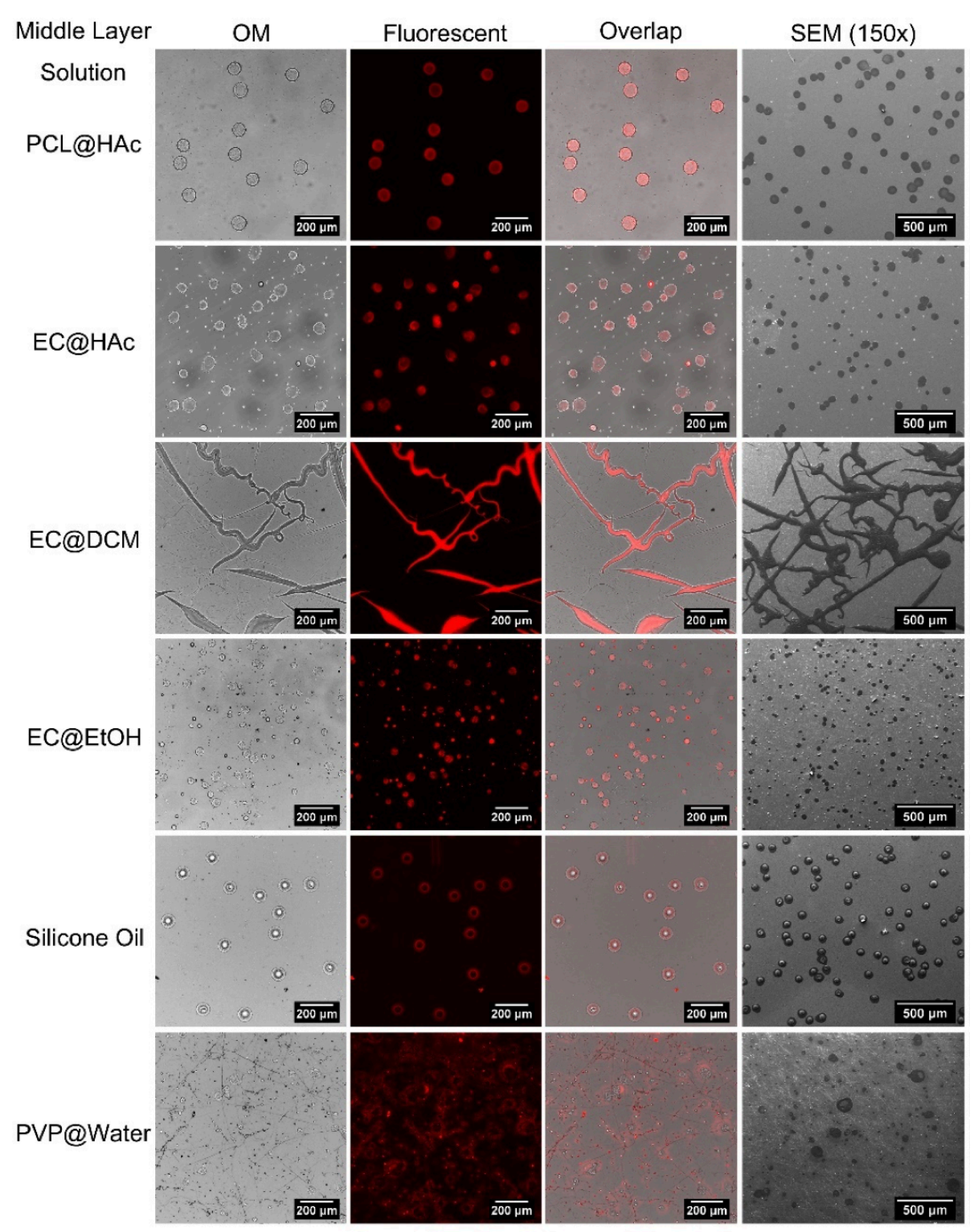

Figure 8. Effect of different intermediate solutions on microparticle formation and surface morphology. 


\section{Conclusion}

This study explores the effects of dominant electrospraying processing parameters on jet formation and stability and particle morphology during tri-axial electrospraying. PCL was selected as the inner and outer layers, while using silicone oil, PVP or ethyl cellulose as the intermediate layer. The results successfully demonstrated that the material properties (polymer type, polymer molecular weight, solution concentration) and processing parameters (flow rate, applied voltage and collector distance) play a significant role in producing spherical microspheres with uniform size distribution. By modifying and regulating these processing parameters, a stable jetting mode can be achieved and multilayered microspheres with predesigned size and morphology can be successfully engineered for an array of applications in the pharmaceutics remit.

\section{Supporting information.}

Digital images of samples.

\section{Acknowledgement}

This work was financially supported by the National Natural Science Foundation of China (No. 81771960) and the Key Science Technologies R\&D Program of Zhejiang Province (2015C02035). 


\section{References}

1. Jenjob, R.; Phakkeeree, T.; Crespy, D., Core-shell particles for drug-delivery, bioimaging, sensing, and tissue engineering. Biomaterials Science 2020, 8, 2756-2770. 2. Barandov, A.; Bartelle, B. B.; Williamson, C. G.; Loucks, E. S.; Lippard, S. J.; Jasanoff, A., Sensing intracellular calcium ions using a manganese-based MRI contrast agent. Nature communications 2019, 10 (1), 1-9.

3. D’Arrigo, G.; Navarro, G.; Di Meo, C.; Matricardi, P.; Torchilin, V., Gellan gum nanohydrogel containing anti-inflammatory and anti-cancer drugs: a multi-drug delivery system for a combination therapy in cancer treatment. European Journal of Pharmaceutics and Biopharmaceutics 2014, 87 (1), 208-216.

4. Villamagna, I. J.; Gordon, T. N.; Hurtig, M. B.; Beier, F.; Gillies, E. R., Poly (ester amide) particles for controlled delivery of celecoxib. Journal of Biomedical Materials Research Part A 2019, 107 (6), 1235-1243.

5. Parhi, P.; Mohanty, C.; Sahoo, S. K., Nanotechnology-based combinational drug delivery: an emerging approach for cancer therapy. Drug discovery today 2012, 17 (17-18), 1044-1052.

6. Narayanan, S.; Pavithran, M.; Viswanath, A.; Narayanan, D.; Mohan, C. C.; Manzoor, K.; Menon, D., Sequentially releasing dual-drug-loaded PLGA-casein core/shell nanomedicine: Design, synthesis, biocompatibility and pharmacokinetics. Acta biomaterialia 2014, 10 (5), 2112-2124.

7. Shin, H.-C.; Alani, A. W.; Rao, D. A.; Rockich, N. C.; Kwon, G. S., Multidrug loaded polymeric micelles for simultaneous delivery of poorly soluble anticancer drugs. Journal of Controlled Release 2009, 140 (3), 294-300.

8. Chang, M.; Stride, E.; Edirisinghe, M., Stimulus-responsive liquids for encapsulation storage and controlled release of drugs from nano-shell capsules. Journal of the royal society interface 2011, 8 (56), 451-456.

9. Gao, Y.; Chang, M.-W.; Ahmad, Z.; Li, J.-S., Magnetic-responsive microparticles with customized porosity for drug delivery. Rsc Advances 2016, 6 (91), 88157-88167.

10. Zhao, D.; Li, J.; Suen, W.; Chang, M.; Huang, J., Preparation and characterization of Ganoderma lucidum spores-loaded alginate microspheres by electrospraying. Materials Science and Engineering: C 2016, 62, 835-842.

11. Cao, Y.; Wang, B.; Wang, Y.; Lou, D., Dual drug release from core-shell nanoparticles with distinct release profiles. Journal of pharmaceutical sciences 2014, 103 (10), 3205-3216.

12. Lee, Y.-H.; Bai, M.-Y.; Chen, D.-R., Multidrug encapsulation by coaxial tricapillary electrospray. Colloids and Surfaces B: Biointerfaces 2011, 82 (1), 104-110.

13. Hu, S.-H.; Chen, Y.-Y.; Liu, T.-C.; Tung, T.-H.; Liu, D.-M.; Chen, S.-Y., Remotely nano-rupturable yolk/shell capsules for magnetically-triggered drug release. Chemical Communications 2011, 47 (6), 1776-1778.

14. Lin, L. S.; Song, J.; Yang, H. H.; Chen, X., Yolk-Shell nanostructures: design, synthesis, and biomedical applications. Advanced Materials 2018, 30 (6), 1704639.

15. Zhang, C.; Li, Y.; Hu, Y.; Peng, Y.; Ahmad, Z.; Li, J.-S.; Chang, M.-W., Porous Yolk-Shell Particle Engineering via Nonsolvent-Assisted Trineedle Coaxial Electrospraying for Burn-Related Wound Healing. ACS applied materials \& interfaces 2019, 11 (8), 7823-7835.

16. Liu, J.; Qiao, S. Z.; Chen, J. S.; Lou, X. W. D.; Xing, X.; Lu, G. Q. M., Yolk/shell nanoparticles: new platforms for nanoreactors, drug delivery and lithium- 
ion batteries. Chemical Communications 2011, 47 (47), 12578-12591.

17. Xie, J.; Wang, C. H., Encapsulation of proteins in biodegradable polymeric microparticles using electrospray in the Taylor cone-jet mode. Biotechnology and bioengineering 2007, 97 (5), 1278-1290.

18. Gao, Y.; Bai, Y.; Zhao, D.; Chang, M.-W.; Ahmad, Z.; Li, J.-S., Tuning microparticle porosity during single needle electrospraying synthesis via a non-solventbased physicochemical approach. Polymers 2015, 7 (12), 2701-2710.

19. Yao, Z.-C.; Gao, Y.; Chang, M.-W.; Ahmad, Z.; Li, J.-S., Regulating polycaprolactone fiber characteristics in-situ during one-step coaxial electrospinning via enveloping liquids. Materials Letters 2016, 183, 202-206.

20. Zhang, C.; Yao, Z.; Ding, Q.; Choi, J.; Ahmad, Z.; Chang, M.-W.; Li, J., The Tri-Needle Coaxial Electrospray Engineering of Magnetic Polymer Yolk-Shell Particles Possessing Dual-Imaging Modality, Multi-Agent Compartments and Trigger Release Potential. ACS Applied Materials \& Interfaces 2017, 9 (25), 21485-21495.

21. Xing, Z.; Zhang, C.; Zhao, C.; Ahmad, Z.; Li, J.-S.; Chang, M.-W., Targeting oxidative stress using tri-needle electrospray engineered Ganoderma lucidum polysaccharide-loaded porous yolk-shell particles. European Journal of Pharmaceutical Sciences 2018, 125, 64-73.

22. Ahmad, Z.; Zhang, H.; Farook, U.; Edirisinghe, M.; Stride, E.; Colombo, P., Generation of multilayered structures for biomedical applications using a novel trineedle coaxial device and electrohydrodynamic flow. Journal of the Royal Society Interface 2008, 5 (27), 1255-1261.

23. Cam, M. E.; Zhang, Y.; Edirisinghe, M., Electrosprayed microparticles: A novel drug delivery method. Taylor \& Francis: 2019.

24. Kim, W.; Kim, S. S., Synthesis of biodegradable triple-layered capsules using a triaxial electrospray method. Polymer 2011, 52 (15), 3325-3336.

25. Zhang, C.; Yao, Z.-C.; Ding, Q.; Choi, J. J.; Ahmad, Z.; Chang, M.-W.; Li, J.-S., Tri-needle coaxial electrospray engineering of magnetic polymer yolk-shell particles possessing dual-imaging modality, multiagent compartments, and trigger release potential. ACS applied materials \& interfaces 2017, 9 (25), 21485-21495.

26. Fordham, S., On the calculation of surface tension from measurements of pendant drops. Proceedings of the Royal Society of London. Series A. Mathematical and Physical Sciences 1948, 194 (1036), 1-16.

27. Lee, B.-B.; Ravindra, P.; Chan, E.-S., New drop weight analysis for surface tension determination of liquids. Colloids and Surfaces A: Physicochemical and Engineering Aspects 2009, 332 (2-3), 112-120.

28. Rosell-Llompart, J.; Grifoll, J.; Loscertales, I. G., Electrosprays in the cone-jet mode: from Taylor cone formation to spray development. Journal of Aerosol Science 2018, 125, 2-31.

29. Loscertales, I. G.; Barrero, A.; Guerrero, I.; Cortijo, R.; Marquez, M.; GananCalvo, A., Micro/nano encapsulation via electrified coaxial liquid jets. Science 2002, 295 (5560), 1695-1698.

30. Lee, S.-H.; Nguyen, X. H.; Ko, H. S., Study on droplet formation with surface tension for electrohydrodynamic inkjet nozzle. Journal of mechanical science and technology 2012, 26 (5), 1403-1408.

31. Sperling, L.; Mishra, V., The current status of interpenetrating polymer networks. Polymers for Advanced Technologies 1996, 7 (4), 197-208.

32. Ewaldz, E.; Brettmann, B., Molecular interactions in electrospinning: from polymer mixtures to supramolecular assemblies. ACS Applied Polymer Materials 2019, 1 (3), 298-308. 
33. Yao, Z.-C.; Jin, L.-J.; Ahmad, Z.; Huang, J.; Chang, M.-W.; Li, J.-S., Ganoderma lucidum polysaccharide loaded sodium alginate micro-particles prepared via electrospraying in controlled deposition environments. International journal of pharmaceutics 2017, 524 (1-2), 148-158.

34. Gao, Y.; Zhao, D.; Chang, M.-W.; Ahmad, Z.; Li, X.; Suo, H.; Li, J.-S., Morphology control of electrosprayed core-shell particles via collection media variation. Materials Letters 2015, 146, 59-64.

35. Gao, Y.; Zhao, D.; Chang, M.-W.; Ahmad, Z.; Li, J.-S., Optimising the shell thickness-to-radius ratio for the fabrication of oil-encapsulated polymeric microspheres. Chemical Engineering Journal 2016, 284, 963-971. 
Table of Content

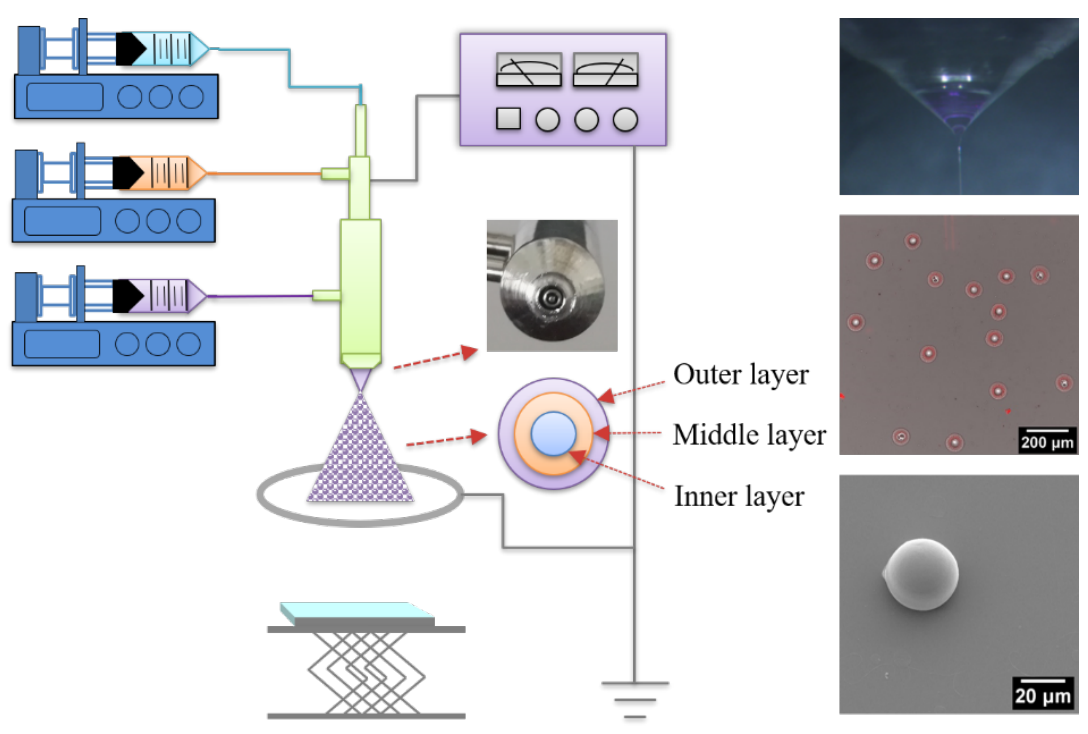

